\title{
Plasmon Resonance of the Hollow Triangular Nanostructures with Different Holes
}

\author{
Leila Sheikhi* and Abbas Azarian \\ Department of Physics, University of Qom, Qom, Iran \\ *Corresponding Author Email: l.sheikhi@stu.qom.ac.ir \\ Regular paper: Received: Sep. 5, 2020, Revised: Oct. 22, 2020, Accepted: Mar. 15, 2021, \\ Available Online: Mar. 17,2021, DOI: 10.29252/ijop.14.2.163
}

\begin{abstract}
In hollow nanostructures, the inner and outer surface plasmons couple together that provides interesting plasmonic properties resulting in the enhancement of the plasmonic field. In addition, triangular structures are interesting for plasmonic applications due to their sharp corners and low symmetry. In this work, gold triangular nanostructures with triangular (TTN) and circular (CTN) holes in their centers have been simulated using the FDTD method and their plasmonic properties have been compared. The extinction spectra of the structures display that CTN has only one plasmonic peak, whereas TTN has several plasmonic modes due to its lower symmetry. Each peak presents different interactions between the multipoles that understand from the charge density distribution. Also, the figure of merit (FOM) of the peaks has been calculated and a high value of 33 is obtained for one of the TTN modes, which is appropriate for sensing application.
\end{abstract}

KEYWORDS: surface plasmon, hollow nanostructure, extinction, charge density, FDTD

\section{I.INTRODUCTION}

Hollow nanostructures have attracted the researchers' attention due to the interesting optical properties resulting from coupling between the inner and outer surface plasmons. Localized surface plasmon resonance (LSPR) is a collective oscillation of the conduction electrons of metallic nanoparticles in response to the incident electromagnetic radiation.
Various factors such as shape, material, size, and the surrounding medium of the nanoparticles affect the plasmon resonance. Besides these factors, the resonance can be adjusted by changing the wall thickness for hollow nanostructures (the effect of wall thickness on the plasmonic resonance of triangular structure has been studied in our previous work [1]) [2]-[4]. However, the plasmon resonance results in the enhancement of the local field. This enhancement is stronger for the hollow nanostructures because of the coupling [4]-[6]. These properties are useful in many applications such as in refractive index sensing, surface enhanced Raman scattering (SERS), fluorescence, plasmonic ruler, and etc. [3]-[4].

The frequency and maximum of the plasmonic peaks are very sensitive to the refractive index of the surrounding medium, making it appropriate for the sensing applications. The ability of the plasmonic peaks for sensing is measured by a quantity called sensitivity (S) that is defined as the ratio of the peaks shift to the refractive index unit $\left(S=\Delta \lambda_{\max } / \mathrm{RIU}\right)$. Moreover, the figure of merit (FOM) is a better quantity for measuring the sensing ability because the detection of the narrower peaks is easier. It is defined as the sensitivity divided to the full width at half maximum (FWHM) of the peaks. Therefore, narrower peaks that have larger FOMs are better for sensing applications [3], [5], [7]. 
Hollow nanostructures with different geometries such as cubic, hexagonal, circular and triangular have been synthesized or numerically simulated [4], [8]-[11]. Galvanic replacement (GR) reactions provide an effective and simple route for generating the metallic hollow nanostructures with scalable hollow interiors and porous walls. For instance, $\mathrm{Ag}-\mathrm{Au}$ alloy triangular nanoframes have been prepared using $\mathrm{Ag}$ nanoprisms as sacrificial templates through a GR reaction with HAuCl4 [12]. Moreover, numerical methods such as discrete dipole approximation (DDA), finite element method (FEM), finite difference time domain (FDTD), and so on have been used for the simulation of the plasmonic properties of the nanostructures. FDTD is a common used method that is simple and versatile. It solves Maxwell equations in the time domain and obtains the absorption and scattering cross-sections and near-field and charge density of the nanostructure [13].

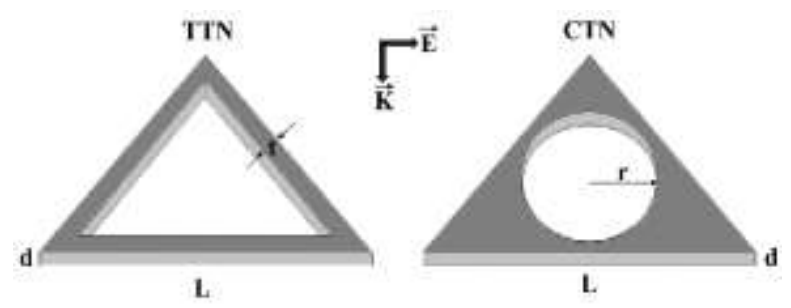

Fig. 1. Schematic of geometric parameters and incident radiation in the simulation of TTN and CTN.

Triangular structures are interesting for plasmonic studies because of the possibility of the exhibiting the high-order plasmonic modes and the electric field enhancement at the sharp corners [14]-[15]. In this work, triangular nanostructures with circular (CTN) and triangular holes (TTN) has been studied using FDTD method and compared their optical properties.

\section{Simulation Method}

The simulation of the optical properties of the nanostructures was performed using the Lumerical FDTD Solutions. The structures were gold equilateral triangles with the edge length (L) of $30 \mathrm{~nm}$ and thickness (d) of $7 \mathrm{~nm}$. Refractive index of gold was taken from the Johnson and Cheristy experimental data. For TTN, a triangular hole with the edge length of $20.8 \mathrm{~nm}$ was placed in the center so that the wall thickness (t) of the structure was $4 \mathrm{~nm}$. Also for CTN, a circular hole of $7 \mathrm{~nm}$ radius (r) was located in the center.

Using a TFSF source, the incident plane wave in the wavelength of $600 \mathrm{~nm}$ to $2000 \mathrm{~nm}$ and polarized in the direction of the $\mathrm{x}$-axis was illuminated in the opposite direction of the $\mathrm{z}$ axis and perpendicular to the plane of the nanostructures ( $x-y$ plane). A schematic of the parameters used in the simulation is shown in Fig. 1. Furthermore, the nanostructure region was discretized by the mesh size of $0.5 \mathrm{~nm}$ in order to obtain high accuracy results. Meanwhile, the boundary conditions of perfectly matched layer (PML) were used to eliminate the additional reflection from the borders.

\section{III.RESULTS AND DISCUSSION}

In order to study and compare the optical properties of two triangular structures, the extinction cross-sections of two similar triangular structures but with two types of circular (CTN) and triangular holes (TTN) were investigated. The extinction spectra of CTN and TTN in a surrounding medium with the refractive index (n) of 1.2 are shown in Fig. 2. CTN has just one plasmonic peak at $902 \mathrm{~nm}$ (BDQ $\mathrm{C}$ mode), while TTN has some plasmonic modes, two main peaks with bigger height at wavelengths of about $701 \mathrm{~nm}$ (mode $\left.\mathrm{BDD}_{\mathrm{T}}\right)$ and $973 \mathrm{~nm}\left(\mathrm{BDQ}_{\mathrm{T}} 2\right.$ mode) (these abbreviations were used by the authors for different plasmon modes according to the type of their interactions which was understood from their charge distribution and explained in the next paragraphs) and several weak peaks (for example at $801 \mathrm{~nm}$ and $832 \mathrm{~nm}$ ) between two peaks.

It is worth to note that the structures with lower symmetry exhibit more plasmon peaks 
possessing higher orders modes [13]-[15]. So, TTN has more peaks due to its lower symmetry compared to CTN. Moreover, depending on the symmetry of the nanostructure, surface plasmon may have a dipole-active character. These surface plasmons are considered as bright modes because they efficiently decay in the far field. Maxwell's equations also predict the existence of dipolar-inactive LSPs: quadrupolar and higher- order eigenmodes cannot interact classically with light and they are generally referred as dark modes. The scattering crosssection of the dark plamon is much smaller than their absorption cross-section. Another plasmonic dark modes are pure near-field modes that can arise from the plasmon hybridization. Dark modes have longer lifetimes in compared to bright modes, due to their lack of a net dipole moment, making them attractive for a number of applications. Moreover, Dark modes are not excited by electromagnetic radiation directly and have the smaller line width than the bright modes [5], [16-17]. Therefore, all the mentioned peaks here are dark modes because the scattering cross-sections of two studied triangular structures are much smaller $\left(10^{-3}\right.$ times) than their adsorption cross-sections.

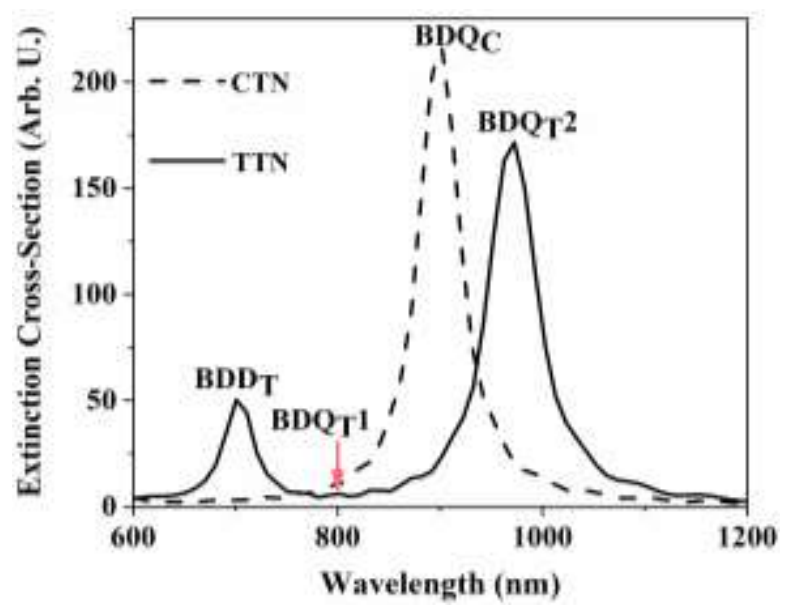

Fig. 2. Extinction spectra of the two triangular structures in a medium with $\mathrm{n}=1.2$. The plasmonic modes of each structure shown on the plot.

The wavelengths of the plasmonic peaks of the structures in the mediums with different refractive index have been plotted in Fig. 3 .
The sensitivity of the plasmon modes obtains from the slope of the line passing through these points. The red shift of the plasmonic peaks with increasing the refractive index of the surrounding medium is seen from the figure. Also, the FOM of the peaks in a medium with $n=1.2$ is shown in Table 1 . For TTN, two main peaks $\left(\mathrm{BDD}_{\mathrm{T}}\right.$ and $\left.\mathrm{BDQ}_{\mathrm{T}} 2\right)$ and one of the small peaks $\left(\mathrm{BDQ}_{\mathrm{T}} 1\right)$ with the wavelength of $801 \mathrm{~nm}$ were considered. FOM of $\mathrm{BDD}_{\mathrm{T}}$ and $\mathrm{BDQ}_{\mathrm{T}} 2$ and $\mathrm{BDQ}_{\mathrm{C}}$ (the peak of CTN) is almost the same (about 12). Whereas, $\mathrm{BDQ}_{\mathrm{T}} 1$ has a high FOM of 33, which is more adequate for sensing application. Interestingly, the sensitivity of this peak and $\mathrm{BDQ}_{\mathrm{C}}$ is almost the same, but the FOM of $\mathrm{BDQ}_{\mathrm{T}} 1$ is about three times larger due to its narrow linewidth (about $18.3 \mathrm{~nm}$ ).

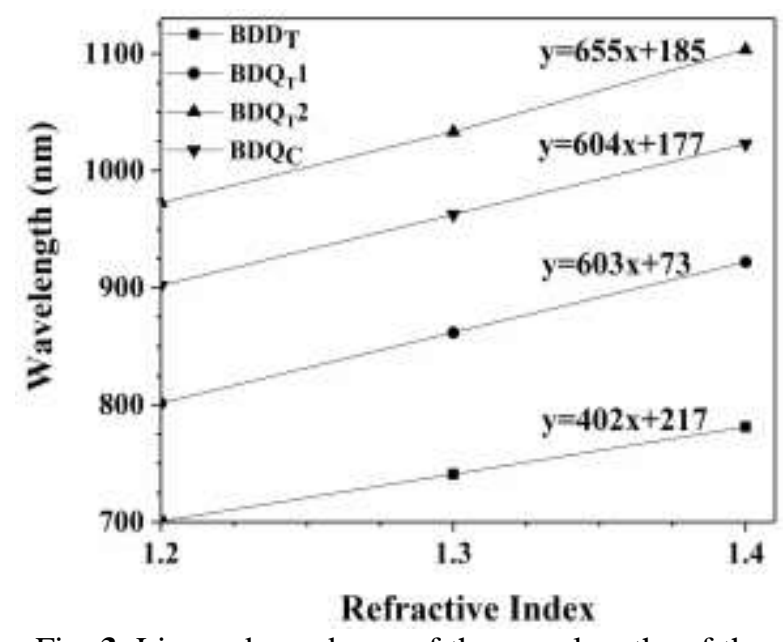

Fig. 3. Linear dependence of the wavelengths of the plasmonic peaks of two triangular structures CTN and TTN to refractive indices of the surrounding medium. The slop of each fitting line is the sensitivity.

In order to distinguish the nature of these peaks, the charge density and the near-field distribution of them were investigated. The charge distribution of the plasmonic peak of CTN (Fig. 4(a)) displays that the two dipoles $\mathrm{P} 1_{\mathrm{C}}$ and $\mathrm{P} 2_{\mathrm{C}}$ in the middle region oscillate antiparallel that almost neutralize each other and form a quadrupole $\left(\mathrm{Q}_{\mathrm{C}}\right)$. This quadrupole interacts with the $\mathrm{P} 3_{\mathrm{C}}$ dipole at the nanostructure bottom and create a bonding (attractive) dipolar-quadrupolar $\left(\mathrm{BDQ}_{\mathrm{C}}\right)$ mode. 
The charge distributions of TTN are shown in Figs. 4(b) to 4(d). As shown for the first peak in Fig. 4(b), dipoles $\mathrm{P} 1_{\mathrm{T}}$ and $\mathrm{P} 2_{\mathrm{T}}$ with Different magnitudes oscillate in opposite directions and create a bonding dipolar-dipolar $\left(\mathrm{BDD}_{\mathrm{T}}\right)$ mode.

Table 1. Magnitude of the FOM of the CTN and TTN modes in a medium with $n=1.2$.

\begin{tabular}{ccccc}
\hline Peak & BDDT & BDQT1 & BDQT2 & BDQC \\
\hline FOM & 11.9 & 33 & 11.5 & 12.3 \\
\hline
\end{tabular}

According to Figs. 4(c) and 4(d), the charge density distribution of the peaks $\mathrm{BDQ}_{\mathrm{T}} 1$ and $\mathrm{BDQ}_{\mathrm{T}} 2$ are similar, but $\mathrm{BDQ}_{\mathrm{T}} 1$ has the larger charge density. So, it has the larger dipoles that possess higher energy, and therefore this mode occurs at the shorter wavelengths. For these peaks, the dipoles of $\mathrm{P}_{\mathrm{T}}$ at the bottom of the triangle and $\mathrm{P} 2{ }_{\mathrm{T}}$ on the two edges oscillate antiparrallel with approximately equal magnitude, forming the $\mathrm{Q}_{\mathrm{T}}$ quadrupole. This quadrupole interacts with the dipole $\mathrm{P} 1_{\mathrm{T}}$ at the apex of the nanoframe and form a bonding dipolar-quadrupolar mode $\left(\mathrm{BDD}_{\mathrm{T}}\right)$.

On the other hand, because the dipole-dipole interaction energy $\left(\mathrm{W}_{\mathrm{DD}}\right)$ is larger than the dipole-quadrupole energy ( $\mathrm{W}_{\mathrm{DQ}}$ ) (according to the formulas of the interaction energy in [18]), $\mathrm{BDD}_{\mathrm{T}}$ mode occurs at higher energies and shorter wavelengths than the other modes of TTN.

The near-field distribution of the plasmon modes is also shown in the inset of Fig. 4. For $\mathrm{BDQ}_{\mathrm{C}}$ mode, the strongest plasmonic field occurs at the corners of the triangle on either side of the base. For all modes of TTN, the maximum field enhancement occurs at the tip of the triangle in the hole region. Moreover, the magnitudes of the field enhancement $\left(\mathrm{E} / \mathrm{E}_{0}\right.$, where $\mathrm{E}_{0}$ is the magnitude of the incident field) at the hotspots of the $\mathrm{BDD}_{\mathrm{T}}, \mathrm{BDQ}_{\mathrm{T}} 1$, and $\mathrm{BDQ}_{\mathrm{T}} 2$ peaks are 70,25 , and 80 , respectively. If the field enhancement is greater, the maximum of the plasmonic peak will be larger [19], so, the height of $\mathrm{BDQ}_{\mathrm{T}} 1$ mode in the extinction spectrum is much smaller than two other peaks.

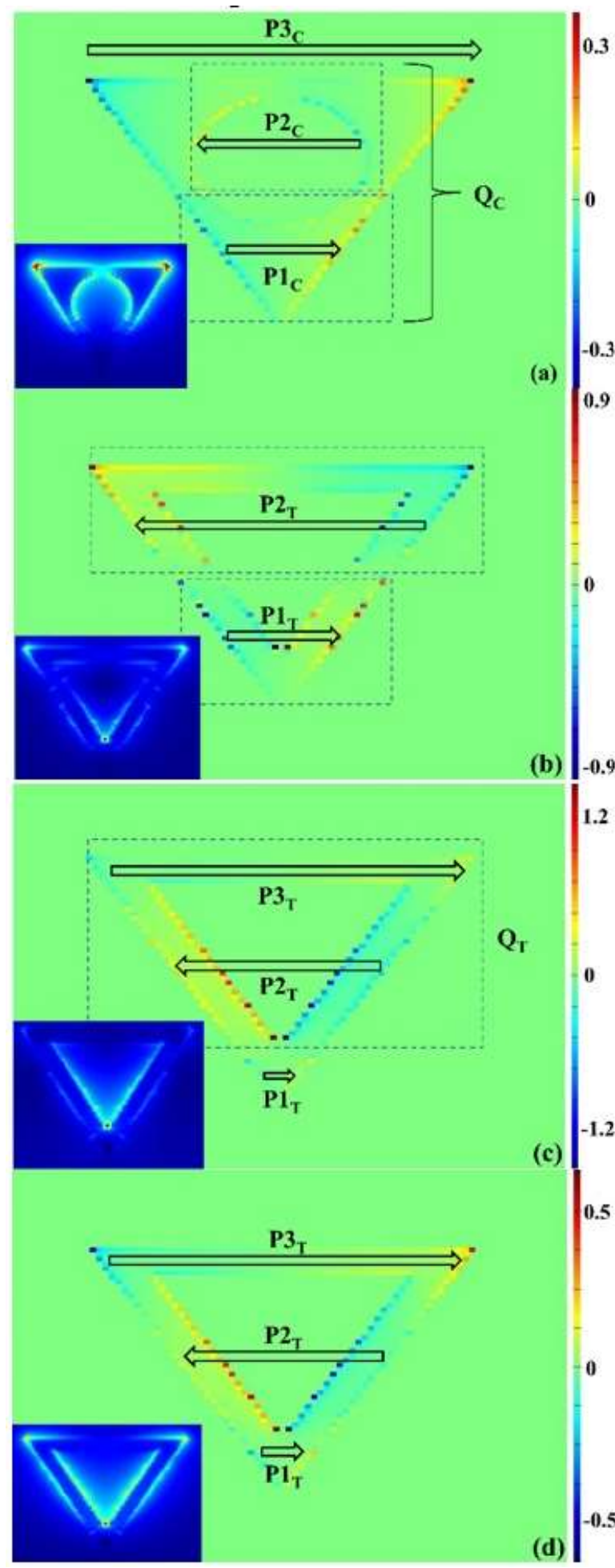

Fig. 4 Charge density distributions of the plasmonic peaks of a) CTN and (b), (c) and (d) TTN. The near-field distribution of the peaks in inset of each part.

\section{IV.CONCLUSION}

Hollow nanostructures have interesting plasmonic properties due to the coupling between the inner and outer surface plasmons. 
In this work, triangular structures with two types of triangular (TTN) and circular (CTN) holes were studied. CTN has just one peak, whereas, for TTN, there are several plasmonic modes because of its lower symmetry. The charge density distributions indicate that the plasmon resonance of CTN is due to the dipolar-quadrupolar interaction and the resonances of TTN is owning to dipolardipolar and dipolar-quadrupolar interactions. The differences of the plasmonic peaks and charge density and near-field distribution of two structures exhibit the effect of the nanostructure shape on the plasmon resonance, even in this case that all parameters were kept the same and only the holes were different.

In addition, a plasmon mode with the FOM of 33 was obtained for TTN, which is much larger than that for CTN (about 12). It is due to its very narrow linewidth (about $18 \mathrm{~nm}$ ) that makes it more suitable for sensing application.

\section{REFERENCES}

[1] A. Azarian and L. Sheikhy, "Dark plasmon with a high figure of merit in a single $\mathrm{Au}$ triangular nano frame," J. Clust. Sci. Vol. 30, pp. 1633-1639, 2019.

[2] R.T. Hill, "Plasmonic biosensors," Wiley interdisciplinary Reviews: Nanomedicine and Nanobiotechnology, Vol. 7, pp. 152-168, 2015.

[3] E. Petryayeva and U.J. Krull, "Localized surface plasmon resonance: nanostructures, bioassays and biosensing-a review," Anal. Chim. Acta, Vol. 706, pp. 8-24, 2011.

[4] M.A. Mahmoud, D. O'Neil, and M.A. ElSayed, "Hollow and solid metallic nanoparticles in sensing and in nanocatalysis," Chem. Mater. Vol. 26, pp. 44-58, 2014.

[5] W. Chen, H. Hu, W. Jiang, Y. Xu, S. Zhang, and $\mathrm{H}$. Xu, "Ultrasensitive nanosensors based on localized surface plasmon resonances: from theory to applications," Chinese Phys. B, Vol. 27, pp. 107403-107425, 2018.

[6] M.A. Mahmoud and M.A. El-Sayed, "Gold nanoframes: very high surface plasmon fields and excellent near-infrared sensors," J. Am.
Chem. SOC. Vol. 132, pp. 12704-12710, 2010.

[7] J. Becker, A. Trügler, A. Jakab, U. Hohenester, and C. Sönnichsen, "The optimal aspect ratio of gold nanorods for plasmonic bio-sensing," Plasmonics, Vol. 5, pp. 161-167, 2010.

[8] Q. Chen, H. Qi, Y.-T. Ren, J.-P. Sun, and L. M. Ruan, "Optical properties of truncated Au nanocages with different size and shape," Aip Adv. Vol. 7, pp. 0651 (15-25), 2017.

[9] B. Hazra and M. Chandra, "Plasmon hybridization mediated structure-specific refractive index sensitivity of hollow gold nanoprism in the vis-NIR region," ACS Sensors, Vol. 1, pp. 536-542, 2016.

[10]C.E. Talley, J.B. Jackson, C. Oubre, N.K. Grady, C.W. Hollars, S.M. Lane, T.R. Huser, P. Nordlander, and N.J. Halas, "Surfaceenhanced Raman scattering from individual $\mathrm{Au}$ nanoparticles and nanoparticle dimer substrates," Nano Lett. Vol. 5, pp. 1569-1574, 2005.

[11]M.M. Shahjamali, M. Bosman, S. Cao, X. Huang, X. Cao, H. Zhang, S.S. Pramana, and C. Xue, "Surfactant- free sub- $2 \mathrm{~nm}$ ultrathin triangular gold nanoframes," Small, Vol. 9, pp. 2880-2886, 2013.

[12] M. Tsuji, T. Kidera, A. Yajima, M. Hamasaki, M. Hattori, Ta. Tsujiab, and H. Kawazumi, "Synthesis of $\mathrm{Ag}-\mathrm{Au}$ and $\mathrm{Ag}-\mathrm{Pd}$ alloy triangular hollow nanoframes by galvanic replacement reactions without and with posttreatment using $\mathrm{NaCl}$ in an aqueous solution," Cryst. Eng. Comm. Vol. 16, pp. 2684-2691, 2014.

[13] V. Amendola, R. Pilot, M. Frasconi, O.M. Maragò, and M.A. Iatì, "Surface plasmon resonance in gold nanoparticles: a review," J. Phys.: Condens. Matter, Vol. 29, pp. 203002203051, 2017.

[14]G. Fletcher, M.D. Arnold, and T. Pedersen, "Multipolar and dark-mode plasmon resonances on drilled silver nano-triangles," Opt. Express, Vol. 23, pp. 18002-18013, 2015.

[15] K. J. Zhang, D. B. Lu, B. Da, and Z. J. Ding, "Coupling of surface plasmon modes and refractive index sensitivity of hollow silver nanoprism," Sci. Rep. Vol. 8, pp. 1-13, 2018. 
[16]S. Zhang and $\mathrm{H} . \mathrm{Xu}$, "Tunable dark plasmons in a metallic nanocube dimer: toward ultimate sensitivity nanoplasmonic sensors," Nanoscale, Vol. 8, pp. 13722-13729, 2016.

[17] M.-W. Chu, V. Myroshnychenko, C.H. Chen, J.-P. Deng, C.-Y. Mou, and F.J.G. Abajo, "Probing bright and dark surface-plasmon modes in individual and coupled noble metal nanoparticles using an electron beam," Nano Lett. Vol. 9, pp. 399-404, 2009.

[18] N. Hooshmand, J. A. Bordley, and M. A. ElSayed, "The sensitivity of the distance dependent plasmonic coupling between two nanocubes to their orientation: edge-to-edge versus face-to-face," J. Phys. Chem. Vol. 120, pp. 4564-4570, 2016.

[19] L.-W. Nien, B. K. Chao, J. Li, and C. H. Hsueh, "Optimized sensitivity and electric field enhancement by controlling localized surface plasmon resonances for bowtie nanoring nanoantenna arrays," Plasmonics, Vol. 10, pp. 553-561, 2015.

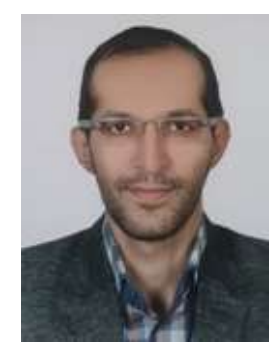

Abbas Azarian graduated in condensed matter physics at the Sharif University of Technology
(SUT), Iran, in 2005. He received his Ph.D. in Nano- optics from the SUT in 2009. He is currently Associate Professor at the Physics Department, University of Qom, Iran. His research activity mainly focuses on the Nano optical sensors.

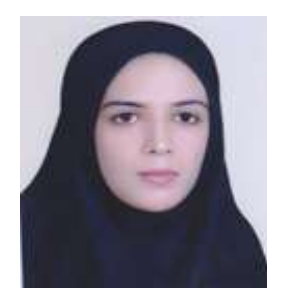

Leila Sheikhi received her BSc degree from Payam Noor University of Jahrom, Iran, in 2007 and her MSc degree from Azzahra University, Iran, in 2012 in solid state physics. The title of her MSc thesis was: "Fabrication and study of the optical-electrical properties of transparent conducting electrodes (TCO) for solar cells from doping $\mathrm{ZnO}$ nano particles". She is currently studying for $\mathrm{PhD}$ degree of solid state physics in University of Qom. 